\title{
Dynamic Panel Probit Models for Current Account Reversals and their Efficient Estimation
}

\begin{abstract}
Nonlinear panel data models have been used to analyze discrete macroeconomic events such as currency crises, sudden stops and current-account reversals. A salient feature of macroeconomic variables to be captured by such models is their distinct serial dependence. We use probit models with state dependence, unobserved heterogeneity, and serially correlated errors in order to uncover the determinants and the dynamics of current-account reversals for a panel of developing and emerging countries. Likelihood evaluation of these models requires high-dimensional interdependent integration. Thus likelihood estimation typically relies upon MonteCarlo (MC) integration techniques. The most popular MC procedure for the evaluation of such choice probabilities is the GewekeHajivassiliou-Keane (GHK) procedure, which has been shown to be innacurate in applications with large time dimension. In the present study we use Efficient Importance Sampling (EIS) to substantially improve the numerical efficiency of the GHK allowing for reliable estimation of dynamic panel probit models. The empirical results suggest that countries with high current account imbalances, low foreign reserves, a small fraction of concessional debt, and unfavorable terms of trades are more likely to experience a current account reversal. Furthermore we find evidence for serially correlated error components and weak evidence of state dependence of the propensity to experience a current-account reversal.
\end{abstract}

JEL classification: C15; C23; C25; F32

Keywords: Panel data, Dynamic discrete choice, Current account reversals, Monte Carlo integration, State dependence. 


\section{Introduction}

The determinants of current account reversals and their consequences for countries' economic performance have received a lot of attention since the currency crises of the 1990s, and have found renewed interest because of the huge current account deficit of the US in recent years. The importance of the current account comes from its interpretation as a restriction on countries' expenditure abilities. Expenditure restrictions, generated by sudden stops and/or currency crises, can generate current account reversals, worsen an economic crises or even trigger one (see, e.g., Milesi-Ferretti and Razin, 1996, 1998, 2000, and Obstfeld and Rogoff, 2004). Typical issues addressed in the recent literature are: The extent to which current account reversals affect economic growth (Milesi-Ferretti and Razin, 2000, and Edwards 2004a,b); The sustainability of large current account deficits for significant periods of time (Milesi-Ferretti and Razin, 2000); and possible causes for current account reversals (Milesi-Ferretti and Razin, 1998, and Edwards, 2004a,b). Our paper proposes to analyze the latter issue in the context of dynamic panel probit models, paying special attention to the serial dependence inherent to current account reversals.

Milesi-Ferretti and Razin (1998) and Edwards (2004a,b) use panel probit models in order to investigate the determinants of current account reversals. While Milesi-Ferretti and Razin analyze a panel of low- and middle-income countries, Edwards also includes industrialized countries. They use time and country specific dummies in order to account for heterogeneity. In addition to the fact that it requires estimation of a large number of parameters, a fixed effect approach raises two key issues of identification in the context of the data set we propose to use. First, it precludes the use of potentially important explanatory variables which are constant across countries or over time. Also, current account crises are typically rare events and have not been experienced by some of the countries included in our data set.

Following Heckman (1981a), Falcetti and Tudela (2006) argue that there are two distinct possible sources of serial dependence which ought to be taken into account in the context of a panel analysis of currency crisis: State dependence and persistent heterogeneity across countries. State dependence would reflect the possibility that past reversals could affect the probability of another reversal. Unobserved heterogeneity would reflect differences in institutional, political or relevant economic factors across countries which cannot be controlled for. However, as argued, e.g., by Hyslop (1999), serial dependence could also be transitory resulting from autocorrelation, whether specific to individual countries (idiosyncratic error component) or common to all (time random effect). Serial dependence in the idiosyncratic error component may arise from a persistence of the current account deficit itself as documented, e.g., by Edwards (2004b).

In the present paper, we analyze the determinants and dynamics of current account reversals for a panel of developing and emerging countries controlling for alternative sources of persistence. Our starting point is a panel probit model with state dependence and persistent random heterogeneity. We then analyze the robustness of this model against the introduction of correlated idiosyncratic 
error components (Section 3.1) or correlated common time effects (Section 3.2).

Likelihood evaluation of panel probit models with unobserved heterogeneity and dynamic error components is complicated by the fact that the computation of the choice probabilities requires high-dimensional interdependent integration. The dimension of such integrals is typically given by the number of time periods $(T)$, or if one allows for interaction between country specific and time random effects by $T+N$, where $N$ is the number of countries. Thus efficient likelihood estimation of such models typically relies upon Monte-Carlo (MC) integration techniques (see, e.g., Geweke and Keane, 2001 and the references therein). Various MC procedures have been proposed for the evaluation of such choice probabilities - see, e.g., Stern (1997) for a survey. The most popular among those is the GHK procedure which was developed by Geweke (1991), Hajivassiliou (1990), and Keane (1994) and which has been applied to the estimation of dynamic panel probit models, e.g., by Hyslop (1999), Greene (2004), and Falcetti and Tudela (2006). While conceptually simple and easy to program, the GHK procedure relies upon importance sampling densities which ignore critical information relative to the underlying dynamic structure of the model. This can lead to significant deterioration of numerical accuracy as the dimensionality of integration increases. In particular, Lee (1997) conducts a MC study of ML estimation under GHK likelihood evaluation for panel models with serially correlated errors and finds significant biases for longer panels.

In the present study we use Efficient Importance Sampling (EIS) methodology developed by Richard and Zhang (2007), which represents a powerful and generic high dimensional simulation technique. It is based on simple LeastSquares optimizations designed to maximize the numerical accuracy of the integral approximations associated with the likelihood. As such, EIS is particularly well suited to handle unobserved heterogeneity and serially correlated errors in panel probit models. In particular, as illustrated below, combining EIS with GHK substantially improves the numerical efficiency of the standard GHK allowing for reliable ML estimation of dynamic panel probit models even in applications with a very large time dimension.

\section{The Data}

Our data set consists of an unbalanced panel for 60 low and middle income countries from Africa, Asia, and Latin America and the Caribbean. The time span of the data set ranges from 1975 to 2004, although the unavailability of some explanatory variables often restrict the analysis to smaller time dimensions. The minimum number of time periods for a country is 9 , the maximum is 18 and the average is 16.5 for a total of 963 observations. The values of the binary dependent variable indicating the occurrence of a current account crisis are known for the initial time period $t=0$ for all countries. Therefore, the initial conditions problem for the estimation of a dynamic discrete choice model including the lagged dependent variable, as discussed, e.g., by Heckman (1981b), does not arise here. The sources of the data are the World Bank's World Development Indicators (2005) and the Global Development Finance 
(2004).

Current account reversal are defined as in Milesi-Ferretti and Razin (1998). According to this definition a current account reversal has to meet three requirements. The first is an average reduction of the current account deficit of at least 3 percentage points of GDP over a period of 3 years relative to the 3 -year average before the event. The second requirement is that the maximum deficit after the reversal must be no larger than the minimum deficit in the 3 years preceding the reversal. The last requirement is that the deficit is reduced to below $10 \%$. The independent variables are standard in the literature and contain lagged macroeconomic, external, debt and foreign variables that are potential indicators of a reversal. The macroeconomic variables are the annual growth rate of GDP (AVGGROW), the share of investment to GDP proxied by the ratio of gross capital formation to GDP (AVGINV), government expenditure (GOV) and interest payments relative to GDP (INTPAY). The external variables are the current account balance as a fraction of GDP (AVGCA), a terms of trade index set equal to 100 for the year 2000 (AVGTT), the share of exports and imports of goods and services to GDP as a measure of trade openness (OPEN), the rate of official transfers to GDP (OT) and the share of foreign exchange reserves to imports (RES). The debt variables included are the share of consessional debt to total debt and interest payments relative to the GDP (CONCDEB). Foreign variables such as the US real interest rate (USINT) and the real growth rates of the OECD countries (GROWOECD) are also included to reflect the influence of the world economy. As in Milesi-Ferretti and Razin (1998), the current account, growth, investment and terms of trade data are 3 -years averages, to ensure consistency with the way reversals are measured.

\section{Empirical Specifications}

The baseline specification we use for our analysis is a dynamic panel probit model of the form

$$
y_{i t}^{*}=x_{i t}^{\prime} \pi+\kappa y_{i t-1}+e_{i t}, \quad y_{i t}=\mathcal{I}\left(y_{i t}^{*}>0\right), \quad i=1, \ldots, N, \quad t=1, \ldots, T,
$$

where $\mathcal{I}\left(y_{i t}^{*}>0\right)$ is an indicator function that transforms the latent continuous variable $y_{i t}^{*}$ for country $i$ in year $t$ into the binary variable $y_{i t}$, indicating the occurrence of a current account reversal. The error term $e_{i t}$ is assumed to be normally distributed with zero mean and a fixed variance. The vector $x_{i t}$ contains the observed macroeconomic, external, debt and foreign variables which might affect the incidence of a reversal. The lagged dependent variable on the right hand side is included to capture possible state dependence. It reflects the possibility that past current account crises could lead to changes in institutional, political or economic factors affecting the probability of another reversal.

\subsection{Panel models with random country-specific effects}

The most restrictive version of the panel probit assumes that the error $e_{i t}$ is independent across time and countries and imposes the restriction $\kappa=0$. This 
produces the standard pooled probit estimator which ignores possible serial dependence and unobserved heterogeneity which cannot be attributed to the variables in $x_{i t}$. However, countries have institutional differences such as property rights, tax systems which are difficult to control for and which might affect their individual propensity to experience a current account reversal. In order to take these differences into account, fixed or random effect panel models could be used. However, a fixed effect model based on country-specific dummy variables, such as the one used in the studies of Milesi-Ferretti and Razin (1998) and Edwards (2004a,b), requires the estimation of a large number of parameters, leading to a significant loss of degrees freedom. Furthermore, since our data set includes countries that never experienced a reversal, for which the dependent variable does not vary, the ML-estimator does not exist. This identification problem restricts the analysis to a random effect approach.

A prominent random effect model is that proposed by Butler and Moffitt (1982). It assumes the following specification for the error term in Equation (1):

$$
e_{i t}=\tau_{i}+\epsilon_{i t}, \quad \epsilon_{i t} \sim \text { i.i.d.N }(0,1), \quad \tau_{i} \sim \text { i.i.d.N }\left(0, \sigma_{\tau}^{2}\right) .
$$

The country-specific term $\tau_{i}$ captures possible permanent latent differences in the propensity to experience a reversal. Furthermore, it is assumed that $\tau_{i}$ and $\epsilon_{i t}$ are independent from the variables included in $x_{i t}$.

Notice that the time-invariant heterogeneity component $\tau_{i}$ implies a crossperiod correlation of the error term $e_{i t}$ which is constant for all pairs of periods and is given by $\operatorname{corr}\left(e_{i t}, e_{i s}\right)=\sigma_{\tau}^{2} /\left(\sigma_{\tau}^{2}+1\right)$ for $t \neq s$ (see, e.g., Greene, 2003). Additional potential sources of serial dependence are transitory country-specific differences in the propensity to experience a reversal leading to serial correlation in the error component $\epsilon_{i t}$ of Equation (1). Furthermore, the intertemporal characteristics of the current account itself (Obstfeld and Rogoff, 1996), and the evidence of sluggish behavior of the trade balance (Baldwin and Krugman, 1989) and of foreign direct investments (Dixit, 1992) might introduce further serial dependence in $\epsilon_{i t}$. Whence, in addition to the Butler-Moffitt specification (1) and (1), we assume here that $e_{i t}$ includes a serially correlated idiosyncratic error component according to

$$
e_{i t}=\tau_{i}+\epsilon_{i t}, \quad \epsilon_{i t}=\rho \epsilon_{i t-1}+\eta_{i t}, \quad \eta_{i t} \sim \text { i.i.d.N }(0,1),
$$

where $\tau_{i}$ and $\eta_{i t}$ are independent among each other and also from the variables included in $x_{i t}$. In order to ensure stationarity we assume that $|\rho|<1$.

\subsection{Panel model with random country- and time-specific effects}

International capital markets, particularly those in emerging economies, appear volatile and subject to spillover effects. The currency crises of the 1990s and the way in which they rapidly spread across emerging markets including those rated as healthy economies by analysts and multilateral institutions, have brought interest in contagion effects (see Edwards and Rigobon, 2002). A crisis in one country may lead investors to withdraw their investments from other markets 
without taking into account differences in economic fundamentals. In addition, a crisis in one economy can also affect the fundamentals of other countries through trade links and currency devaluations. Trading partners of a country in which a financial crisis has induced a sharp currency depreciation could experience a deterioration of the trade balance resulting from a decline in exports and an increase in imports (see Corsetti et al., 1999). These effects can lead to a deterioration of the current account in other countries. In the words of the former Managing Director of the IMF: "from the viewpoint of the international system, the devaluations in Asia will lead to large current account surpluses in those countries, damaging the competitive position of other countries and requiring them to run current account deficit." Fisher (1998).

Currency devaluations of countries that experience a crisis can often be seen as a beggar-thy-neighbor policy in the sense that they incite output growth and employment domestically at the expense of output growth, employment and current account deficit abroad (Corsetti et al., 1999). Competitive devaluations also happen in response to this process, as other economies may try to avoid this competitiveness loss through a devaluation of their own currency. This appears to have happened during the East Asian crises in 1997 (Dornbusch et al., 2000).

The panel probit models introduced above do not account for such spillover effects since they ignore correlation across countries. In order to address this issue we also consider the following factor specification for the error $e_{i t}$ in the probit regression (1):

$$
e_{i t}=\tau_{i}+\xi_{t}+\epsilon_{i t}, \quad \epsilon_{i t} \sim \text { i.i.d.N }(0,1),
$$

with

$$
\xi_{t}=\delta \xi_{t-1}+\nu_{t}, \quad \nu_{t} \sim \text { i.i.d.N }\left(0, \sigma_{\xi}^{2}\right),
$$

where $\tau_{i}, \epsilon_{i t}$ and $\nu_{t}$ are mutually independent and independent from $x_{i t}$. Furthermore, it is assumed that $|\delta|<1$. The common dynamic factor $\xi_{t}$ represents unobserved time-specific effects which induce correlation across countries, reflecting possible spillover effects.

\section{Maximum-Likelihood Estimation}

ML estimation of the simple pooled panel probit model is straightforward and essentially the same as for a single equation probit model. Efficient parameter estimates can also be easily obtained for the Butler-Moffitt model (1) and (1). In particular, the choice probabilities are represented by one dimensional integrals, which can be evaluated conveniently by means of a quadrature procedure. Let $y=\left\{\left\{y_{i t}\right\}_{t=1}^{T}\right\}_{i=1}^{N}$ and $\underline{x}=\left\{\left\{x_{i t}\right\}_{t=1}^{T}\right\}_{i=1}^{N}$. Let $\theta$ denote the parameter vector to be estimated. The likelihood function for the Butler-Moffitt random effect model is then given by

$$
L(\theta ; \underline{y}, \underline{x})=\prod_{i=1}^{N}\left\{\int_{\mathbb{R}^{1}} \prod_{t=1}^{T}\left[\Phi_{i t}^{y_{i t}}\left(1-\Phi_{i t}\right)^{\left(1-y_{i t}\right)}\right] \frac{1}{\sqrt{2 \pi \sigma_{\tau}^{2}}} \exp \left\{\frac{-\tau_{i}^{2}}{2 \sigma_{\tau}^{2}}\right\} d \tau_{i}\right\},
$$


where $\Phi_{i t}=\Phi\left(x_{i t}^{\prime} \pi+\kappa y_{i t-1}+\tau_{i}\right)$, and $\Phi(\cdot)$ represents the cdf of the standardized normal distribution. In the application below, the one dimensional integrals in $\tau_{i}$ are evaluated using a Gauss-Hermite quadrature rule (see, e.g., Butler and Moffitt, 1982).

In contrast to the Butler-Moffitt model, the computation of the likelihood for the model (1) and (2) with country-specific effects and a serially correlated idiosyncratic error component requires the evaluation of $(T+1)$-dimensional interdependent integrals, and that of the model (1), (3), and (4) with countryspecific and time effects the evaluation of $(T+N)$-dimensional integrals. Efficient estimation of these models cannot be obtained by means of standard numerical integration procedures. Instead, we propose to use the EIS methodology of Richard and Zhang (2007). EIS is a highly accurate MC integration procedure developed for the evaluation of high-dimensional integrals and is, therefore, ideally suited for ML estimation of non-linear panel models with unobserved random heterogeneity and serially correlated errors.

In the following two subsections we provide a brief description of the EIS implementation in the context of ML estimation of the panel probit model with random country-specific effects and serially correlated idiosyncratic errors (subsection 4.1), and with random country-specific and time effects (subsection 4.2). For the general theory of EIS, see Richard and Zhang (2007).

\subsection{ML-EIS for random country-specific effects and serially cor- related errors}

The likelihood function of the panel probit model defined by Equations (1) and (2) has the form $L(\theta ; \underline{y}, \underline{x})=\prod_{i=1}^{N} I_{i}(\theta)$, where $I_{i}$ represents the likelihood contribution of country $i$. In the following, we derive the likelihood function for a single country, deleting the subscript $i$ for the ease of notation. Let $\underline{\lambda}^{\prime}=\left(\tau, \epsilon_{1}, \ldots, \epsilon_{T}\right)$ and $\mu_{t}=x_{t}^{\prime} \pi+\kappa y_{t-1}$. Under the assumption that $\epsilon_{0}=0$ the likelihood function is given by

$$
I(\theta)=\int_{\mathbb{R}^{T+1}} \prod_{t=1}^{T} \varphi_{t}\left(\underline{\lambda}_{t}\right) f_{\tau}(\tau) d \underline{\lambda},
$$

where $\underline{\lambda}_{t}^{\prime}=\left(\epsilon_{t}, \epsilon_{t-1}, \tau\right), \underline{\lambda}_{1}^{\prime}=\left(\epsilon_{1}, \tau\right)$ and

$$
\begin{aligned}
\varphi_{t}\left(\underline{\lambda}_{t}\right) & = \begin{cases}\mathcal{I}\left(\epsilon_{t} \in D_{t}\right) \phi\left(\epsilon_{t}-\rho \epsilon_{t-1}\right), & \text { if } t>1 \\
\mathcal{I}\left(\epsilon_{1} \in D_{1}\right) \phi\left(\epsilon_{1}\right), & \text { if } t=1\end{cases} \\
D_{t} & =\left\{\begin{array}{lll}
{\left[-\left(\mu_{t}+\tau\right), \infty\right),} & \text { if } & y_{t}=1 \\
\left(-\infty,-\left(\mu_{t}+\tau\right)\right], & \text { if } & y_{t}=0 .
\end{array}\right.
\end{aligned}
$$

$\mathcal{I}(\cdot)$ denotes the indicator function and $\phi(\cdot)$ the standardized Normal density. $I(\theta)$ will be evaluated by Importance Sampling under trajectories $\left\{\tilde{\tilde{\lambda}}^{(j)}\right\}_{j=1}^{S}$ drawn from an Efficient Importance Sampling density $m(\underline{\lambda} \mid \cdot)$ constructed as described in Richard and Zhang (2007). In order to simplify the application of sequential EIS to the integral in (6) it proves convenient to relabel $\tau$ as $\lambda_{0}$ and 
to rewrite the integral as

$$
I(\theta)=\int_{\mathbb{R}^{T+1}} \prod_{t=0}^{T} \varphi_{t}\left(\underline{\lambda}_{t}\right) d \underline{\lambda},
$$

where $\varphi_{0}\left(\lambda_{0}\right)=f_{\tau}(\tau)$. Also we partition $\underline{\lambda}_{t}^{\prime}$ into $\left(\epsilon_{t}, \underline{\eta}_{t-1}^{\prime}\right)$ with $\underline{\eta}_{t-1}^{\prime}=\left(\epsilon_{t-1}, \lambda_{0}\right)$ for $t>1, \eta_{0}=\lambda_{0}$ and $\eta_{-1}=\emptyset$. EIS aims at constructing a sequence of auxiliary importance samplers of the form

$$
m_{t}\left(\epsilon_{t} \mid \underline{\eta}_{t-1} ; a_{t}\right)=\frac{k_{t}\left(\underline{\lambda}_{t} ; a_{t}\right)}{\chi_{t}\left(\underline{\eta}_{t-1} ; a_{t}\right)}, \quad t=0, \ldots ., T
$$

with

$$
\chi_{t}\left(\underline{\eta}_{t-1} ; a_{t}\right)=\int_{\mathbb{R}} k_{t}\left(\underline{\lambda}_{t} ; a_{t}\right) d \epsilon_{t},
$$

where $\left\{k_{t}\left(\underline{\lambda}_{t} ; a_{t}\right) ; a_{t} \in A_{t}\right\}$ denote a (pre-selected) class of auxiliary parametric density kernels with $\chi_{t}\left(\underline{\eta}_{t-1} ; a_{t}\right)$ as analytical integrating factor in $\epsilon_{t}$ given $\left(\underline{\eta}_{t-1}, a_{t}\right)$. The integral in $(9)$ is rewritten as

$$
I(\theta)=\chi_{0}\left(a_{0}\right) \int_{\mathbb{R}^{T+1}} \prod_{t=0}^{T}\left[\frac{\varphi_{t}\left(\underline{\lambda}_{t}\right) \chi_{t+1}\left(\underline{\eta}_{t} ; a_{t+1}\right)}{k_{t}\left(\underline{\lambda}_{t} ; a_{t}\right)}\right] m_{t}\left(\epsilon_{t} \mid \underline{\eta}_{t-1} ; a_{t}\right) d \underline{\lambda}
$$

with $\chi_{T+1}(\cdot) \equiv 1$. The backward transfer of the integrating factors $\chi_{t+1}(\cdot)$ constitutes the cornerstone of sequential EIS and is meant to capture as closely as possible the dynamics of the underlying process. As discussed further below, it is precisely the lack of such transfers which explains the inefficiency of the GHK procedure in (large dimensional) interdependent truncated integrals. Under (12), an EIS-MC estimate of $I(\theta)$ is given by

$$
\bar{I}_{S}(\theta)=\chi_{0}\left(a_{0}\right) \frac{1}{S} \sum_{j=1}^{S}\left[\prod_{t=0}^{T} \frac{\varphi_{t}\left(\tilde{\hat{\lambda}}_{t}^{(j)}\right) \chi_{t+1}\left(\underline{\tilde{q}}_{t}^{(j)} ; a_{t+1}\right)}{k_{t}\left(\underline{\tilde{\lambda}}_{t}^{(j)} ; a_{t}\right)}\right],
$$

where $\left\{\underline{\tilde{\lambda}}^{(j)}=\left\{\underline{\tilde{\lambda}}_{t}^{(j)}\right\}_{t=0}^{T}\right\}_{j=1}^{S}$ denote $S$ i.i.d. trajectories drawn from the auxiliary sampler

$$
m(\underline{\lambda} \mid a)=\prod_{t=0}^{T} m_{t}\left(\epsilon_{t} \mid \underline{\eta}_{t-1} ; a_{t}\right), \quad a=\left(a_{0}, \ldots, a_{T}\right) \in A=\times_{t=0}^{T} A_{t} .
$$

That is to say, $\tilde{\epsilon}_{t}^{(j)}$ is drawn from $m_{t}\left(\epsilon_{t} \mid \tilde{\eta}_{t-1}^{(j)} ; a_{t}\right)$ for $t=0, \ldots, T$. An Efficient Importance Sampler is one which minimizes the MC sampling variances of the ratios $\varphi_{t} \chi_{t+1} / k_{t}$ under such draws. Since $m_{t}\left(\cdot ; a_{t}\right)$ depends itself upon $a_{t}$, efficient $a_{t}$ 's obtain as solutions of the following fixed point sequences of back-recursive auxiliary least squares (LS) problems:

$$
\begin{gathered}
\left(\hat{c}_{t}^{(k+1)}, \hat{a}_{t}^{(k+1)}\right)=\arg \min _{c_{t}, a_{t}} \sum_{j=1}^{S}\left\{\ln \left[\varphi_{t}\left(\tilde{\tilde{\lambda}}_{t}^{(k, j)}\right) \cdot \chi_{t+1}\left(\underline{\tilde{\eta}}_{t}^{(k, j)} ; \hat{a}_{t+1}^{(k+1)}\right)\right]\right. \\
\left.-c_{t}-\ln k_{t}\left(\tilde{\tilde{\lambda}}_{t}^{(k, j)} ; a_{t}\right)\right\}^{2}
\end{gathered}
$$


for $t=T, T-1, \ldots, 0$, where $\left\{\underline{\tilde{\lambda}}^{(k, j)}=\left\{\underline{\tilde{\lambda}}_{t}^{(k, j)}\right\}_{t=0}^{T}\right\}_{j=1}^{S}$ denote trajectories drawn from $m\left(\underline{\lambda} \mid \hat{a}^{(k)}\right)$. Convergence to a fixed point solution typically requires 3 to 5 iterations for reasonably well-behaved applications. See Richard and Zhang (2007) for details. As starting values we propose to use those values for the auxiliary parameters $a$ implied by the GHK sampling densities discussed further below.

Two additional key components of this EIS algorithm are as follows: (i) The kernel $k_{t}\left(\underline{\lambda}_{t} ; a_{t}\right)$ has to approximate the ratio $\varphi_{t}\left(\underline{\lambda}_{t}\right) \cdot \chi_{t+1}\left(\underline{\eta}_{t} ; a_{t+1}\right)$ with respect to $\underline{\lambda}_{t}$, not just $\epsilon_{t}$ in order to capture the interdependence across the $\epsilon_{t}$ 's. (There is no revisiting of period $t$ once $\hat{a}_{t}$ has been found); (ii) All trajectories $\left\{\underline{\tilde{\lambda}}^{(k, j)}\right\}_{j=1}^{S}$ have to be obtained by transformation of a single set of Common Random Numbers (CRNs) $\left\{\underline{\tilde{u}}^{(j)}\right\}_{j=1}^{S}$ pre-drawn from a canonical distribution, i.e. one which does not depend on $a$. In the present case the CRNs consist of draws from a uniform distribution to be transformed into (truncated) gaussian draws from $m_{t}\left(\epsilon_{t} \mid \underline{\tilde{\eta}}_{t-1} ; \hat{a}_{t}\right)$ (see Appendix 1).

Next, we discuss the specific application of EIS to the likelihood integral defined by Equation (6). Note first that the period- $t$ integrand in Equation (6) includes a (truncated) gaussian kernel. Therefore, it appears appropriate to select a gaussian kernel for $k_{t}\left(\underline{\lambda}_{t} ; a_{t}\right)$, a choice further supported by the fact that we shall demonstrate that $\chi_{t}\left(\underline{\eta}_{t} ; a_{t}\right)$ then takes the form of a gaussian kernel times a probability. Moreover, the selection of a gaussian kernel enables us to take full advantage of the fact that the class of gaussian kernels in $\underline{\lambda}_{t}$ is closed under multiplication (see DeGroot, 1970). Therefore, we specify $k_{t}$ as the following product

$$
k_{t}\left(\underline{\lambda}_{t} ; a_{t}\right)=\varphi_{t}\left(\underline{\lambda}_{t}\right) \cdot k_{0, t}\left(\underline{\lambda}_{t} ; a_{t}\right),
$$

where $k_{0, t}$ is itself a gaussian kernel in $\underline{\lambda}_{t}$. It immediately follows that $\varphi_{t}$. $\chi_{t+1} / k_{t} \equiv \chi_{t+1} / k_{0, t}$ so that $\varphi_{t}$ cancels out in the auxiliary EIS-LS optimization problem as defined in Equation (15). Under specification (16), we follow the standard EIS implementation as described above, but need to pay attention to the fact that $\lambda_{0}=\tau$ is present in all $T+1$ factors of the integrand. Whence, we proceed as follows:

(i) We regroup all terms in $k_{t}$ which only depend on $\lambda_{0}$. Let denote the corresponding factorization as

$$
k_{t}\left(\underline{\lambda}_{t} ; a_{t}\right)=k_{1, t}\left(\underline{\lambda}_{t} ; a_{t}\right) \cdot k_{3, t}\left(\lambda_{0} ; a_{t}\right) ;
$$

(ii) Let $\chi_{1, t}$ denote the integral of $k_{1, t}$ w.r.t. $\epsilon_{t}$ such that

$$
\chi_{1, t}\left(\underline{\eta}_{t-1} ; a_{t}\right)=\int_{\mathbb{R}} k_{1, t}\left(\underline{\lambda}_{t} ; a_{t}\right) d \epsilon_{t} .
$$

Note that this integral is truncated to $D_{t}$ due to the indicator function $\mathcal{I}\left(\epsilon_{t} \in\right.$ $D_{t}$ ) which is included in $\varphi_{t}$ and, therefore, in $k_{1, t}$. Since $k_{1, t}$ is symmetric in $\epsilon_{t}$, the (conditional) probability that $\epsilon_{t} \in D_{t}$ is the same as that of $\epsilon_{t} \in D_{t}^{*}$, where

$$
D_{t}^{*}=\left(-\infty, \gamma_{t}+\delta_{t} \lambda_{0}\right], \quad \text { with } \quad \gamma_{t}=\left(2 y_{t}-1\right) \mu_{t}, \quad \delta_{t}=\left(2 y_{t}-1\right) .
$$


It follows that $\chi_{1, t}$ takes the form of a gaussian kernel in $\underline{\eta}_{t-1}$ times the probability that $\epsilon_{t} \in D_{t}^{*}$ conditional on $\underline{\eta}_{t-1}$, say

$$
\chi_{1, t}\left(\underline{\eta}_{t-1} ; a_{t}\right)=\Phi\left(\alpha_{t}+\beta_{t}^{\prime} \underline{\eta}_{t-1}\right) \cdot k_{2, t}\left(\underline{\eta}_{t-1} ; a_{t}\right),
$$

where $\left(\alpha_{t}, \beta_{t}^{\prime}\right)$ are appropriate functions of $a_{t}$ and the data.

It follows from Equations (17) to (20) that the integral of $k_{t}$ w.r.t. $\epsilon_{t}$ is of the form

$$
\chi_{t}\left(\underline{\eta}_{t-1} ; a_{t}\right)=k_{3, t}\left(\lambda_{0} ; a_{t}\right)\left[\Phi\left(\alpha_{t}+\beta_{t}^{\prime} \underline{\eta}_{t-1}\right) \cdot k_{2, t}\left(\underline{\eta}_{t-1} ; a_{t}\right)\right] .
$$

In direct application of the backward transfer of integrating factors associated with sequential EIS, the factor $k_{3, t}$ is transferred back directly into the period $t=0$ integral while the two factors between brackets are transferred back into the period $t-1$ integral. Full details are provided in Appendix 1.

We conclude this heuristic presentation of the EIS-application to the panel probit model defined by Equations (1) and (2) with two important comments. Firstly, as mentioned above, the MC procedure most frequently used to compute choice probabilities is the GHK technique. It is also an importance sampling procedure but it selects $\varphi_{t}$ itself as the auxiliary period- $t$ kernel. The corresponding importance sampling density is given by

$$
m_{t}\left(\epsilon_{t} \mid \underline{\eta}_{t-1}\right)=\frac{\varphi_{t}\left(\underline{\lambda}_{t}\right)}{\Phi\left(\gamma_{t}+\delta_{t} \lambda_{0}+\delta_{t} \rho \epsilon_{t-1}\right)}, \quad t=1, \ldots, T,
$$

and $m_{0}\left(\lambda_{0}\right)=f_{\tau}(\tau)$ for period $t=0$. The GHK-MC estimate of $I(\theta)$ is then obtained as

$$
\bar{I}_{S}(\theta)=\frac{1}{S} \sum_{j=1}^{S}\left[\prod_{t=1}^{T} \Phi\left(\gamma_{t}+\delta_{t} \tilde{\lambda}_{0}^{(j)}+\delta_{t} \rho \tilde{\epsilon}_{t-1}^{(j)}\right)\right],
$$

where $\left\{\tilde{\tilde{\lambda}}^{(j)}\right\}_{j=1}^{S}$ denotes i.i.d. trajectories drawn from the sequential samplers $\left(m_{0}\left(\lambda_{0}\right),\left\{m_{t}\left(\epsilon_{t} \mid \underline{\eta}_{t-1}\right)\right\}_{t=1}^{T}\right)$. Note that the GHK importance sampler actually belongs to the class of auxiliary EIS samplers introduced in Equation (16) since it amounts to selecting a diffuse $k_{0, t} \propto 1$. Therefore, it is inefficient within this class.

\subsection{ML-EIS for random country-specific and time effects}

The likelihood function for the random effect panel model consisting of Equations (1), (3), and (4) is given by

$$
L(\theta ; \underline{y}, \underline{x})=\int_{\mathbb{R}^{T+N}} \prod_{i=1}^{N} \prod_{t=1}^{T}\left[\Phi\left(z_{i t}\right)\right]^{y_{i t}}\left[1-\Phi\left(z_{i t}\right)\right]^{\left(1-y_{i t}\right)} p(\underline{\tau}, \underline{\xi}) d \underline{\tau}, d \underline{\xi},
$$

with $\underline{\xi}=\left\{\xi_{t}\right\}_{t=1}^{T}, \underline{\tau}=\left\{\tau_{i}\right\}_{i=1}^{N}$ and $z_{i t}=x_{i t}^{\prime} \pi+\kappa y_{i t-1}+\tau_{i}+\xi_{t}$. The presence of a time effect $\xi_{t}$ common to all countries prevents us from factorizing the likelihood function into a product of integrals for each individual country. We 
assume that the $\tau_{i}$ 's are independent across countries but allow for correlation among $\underline{\xi}$. Whence, the joint density of $(\underline{\tau}, \underline{\xi})$ is assumed to be proportional to

$$
p(\underline{\tau}, \underline{\xi}) \propto \sigma_{\tau}^{-N} \exp \left\{-\frac{1}{2 \sigma_{\tau}^{2}} \tau_{i}^{2}\right\}\left|H_{\xi}\right|^{\frac{1}{2}} \exp \left\{-\frac{1}{2} \underline{\xi^{\prime}} H_{\xi} \underline{\xi}\right\},
$$

where $H_{\xi}$ denotes the precision matrix of $\xi$. See Richard (1977) for analytical expressions of $H_{\xi}$ under alternative initial conditions, including stationarity. Conditionally on $\underline{\xi}$, one could apply GHK to each country individually, though Gauss-Hermite would likely be more efficient for these univariate integrals in $\tau_{i}$. One would then be left with a complicated $T$-dimensional integral in $\xi$. In contrast, EIS can be applied to the likelihood function (24) in a way which effectively captures the complex interdependence between $\underline{\tau}$ and $\underline{\xi}$. We shall just outline the main steps of this EIS implementation. See Richard and Zhang (2007) or Liesenfeld and Richard (2007) for details.

The integrand in Equation (24) is first factorized as follows

$$
L(\theta ; \underline{y}, \underline{x})=\int_{\mathbb{R}^{T+N}} \phi_{0}(\underline{\xi}) \prod_{i=1}^{N} \phi_{i}\left(\tau_{i}, \underline{\xi}\right) d \underline{\tau} d \underline{\xi},
$$

where

$$
\begin{aligned}
\phi_{0}(\underline{\xi}) & \propto\left|H_{\xi}\right|^{\frac{1}{2}} \exp \left[-\frac{1}{2} \underline{\xi}^{\prime} H_{\xi} \underline{\xi}\right] \\
\phi_{i}\left(\tau_{i}, \underline{\xi}\right) & \propto \sigma_{\tau}^{-1} \exp \left[-\frac{1}{2 \sigma_{\tau}^{2}} \tau_{i}^{2}\right] \prod_{t=1}^{T}\left[\Phi\left(z_{i t}\right)\right]^{y_{i t}}\left[1-\Phi\left(z_{i t}\right)\right]^{\left(1-y_{i t}\right)} .
\end{aligned}
$$

It is critical that the EIS sampler $m(\underline{\tau}, \underline{\xi} ; a)$ fully reflects the interdependence structure of the posterior density of $(\underline{\tau}, \underline{\xi})$ which is proportional to the integrand in Equation (26). Specifically, the $\bar{\tau}_{i}$ 's are independent from one another conditionally on $\underline{\xi}$ but are individually linked to the full $\underline{\xi}$-vector. Accordingly, the auxiliary sampler is factorized as

$$
m(\underline{\tau}, \underline{\xi} ; a)=m_{0}\left(\underline{\xi} ; a_{0}\right) \prod_{i=1}^{N} m_{i}\left(\tau_{i} \mid \underline{\xi} ; a_{i}\right) .
$$

The corresponding kernels $\left\{k_{i}\left(\tau_{i}, \xi ; a_{i}\right)\right\}_{i=1}^{N}$ and $k_{0}\left(\xi ; a_{0}\right)$ are specified as joint gaussian kernels in $\left(\tau_{i}, \underline{\xi}\right)$ and $\underline{\xi}$, respectively. Significant simplifications follow from the particular form of the integrand in Equation (26). First, note that $\ln \phi_{i}$ is given by

$$
\ln \phi_{i}\left(\tau_{i}, \underline{\xi}\right) \propto-\frac{1}{2} \frac{\tau_{i}^{2}}{\sigma_{\tau}^{2}}+\sum_{t=1}^{T} \ln \left\{\left[\Phi\left(z_{i t}\right)\right]^{y_{i t}}\left[1-\Phi\left(z_{i t}\right)\right]^{\left(1-y_{i t}\right)}\right\} .
$$

Each factor in the sum depends only on a single $z_{i t}$. Therefore, $\ln k_{i}$ is specified as follows

$$
\ln k_{i}\left(\tau_{i}, \underline{\xi} ; a_{i}\right)=-\frac{1}{2}\left[\frac{\tau_{i}^{2}}{\sigma_{\tau}^{2}}+\sum_{t=1}^{T}\left(\alpha_{i, t} z_{i, t}^{2}+2 \beta_{i, t} z_{i, t}\right)\right]
$$


for a total of $2 \cdot T$ auxiliary parameters plus the intercept. It follows that, at the cost of standard algebraic operations $\chi_{i}\left(\xi ; a_{i}\right)$ (i.e. the integrating constant for $\left.k_{i}\right)$ is itself a gaussian kernel in $\underline{\xi}$. Whence, the product $\phi_{0}(\xi) \cdot \prod_{i=1}^{N} \chi_{i}\left(\xi ; a_{i}\right)$ is a gaussian kernel and requires no further adjustment (an interesting example of perfect fit in an EIS auxiliary regression).

\section{Empirical Results}

The ML estimate of the pooled probit model (1) under the assumption that the errors are independent across time and countries are presented in Table 2. The results for the static model $(\kappa=0)$ are reported in the left columns and those of the dynamic specification including the lagged dependent in the right columns.

The parameter estimates are all in line with the results in the empirical literature on current account crises (see Milesi-Ferretti and Razin, 1998, and Edwards 2004a,b). Sharp reductions of the current-account deficit are more likely in countries with a high current account deficits (AVGCA) and with higher government expenditures (GOV). The significant effect of the current account deficit level is consistent with a need for sharp corrections in the trade balance to ensure that the country remains solvent. Interpreting current account as a constraint on expenditures, the positive impact of government expenditure on the reversal probability can be attributed to fact that an increase of government expenditures leads to a deterioration of the current account. However, the inclusion of the lagged dependent variable reduces this effect and makes it non significant. This suggests that government expenditures might capture some omitted serial dependence under the static specification. The coefficient of foreign reserve (RES) is negative and significant which suggests that low levels of reserves make it more difficult to sustain a large trade imbalance and may also reduce foreign investors' willingness to lend (Milesi-Ferretti and Razin, 1998). Also, reversals seem to be less common in countries with a high share of concessional debt (CONCDEB). This would be consistent with the fact that concessional debts tend to be higher in countries which have difficulties reducing external imbalances. Finally, countries with weaker terms of trade (AVGTT) and higher GDP growth (AVGGROW) seem to face higher probabilities of reversals, especially when growth rate in OECD countries (GROWOECD) and/or US interest rate (USINT) are higher - though none of these four coefficients are statistically significant.

The inclusion of the lagged current account reversal variable substantially improves the fit of the model as indicated by the highly significant increase of the maximized log-likelihood value. The estimated coefficient $\kappa$ measuring the impact of the lagged dependent state variable is positive and significant at the $1 \%$ significance level. This suggests that a current account reversal significantly increases the probability of a further reversal the following year. But as explained above, country-specific differences generate some source of autocorrelation in the error term that will be captured by the state dependence parameter if not accounted for, leading to wrong conclusions about state dependence. 
Table 3 reports the estimates of the Butler-Moffitt model (1) and (1), which includes random country specific effects, leading to equicorrelated errors across time periods. The ML-estimates are obtained using a 20-points Gauss Hermite quadrature. The estimate of the coefficient $\sigma_{\tau}$ indicates that only $3 \%$ of the total variation in the latent error is due to unobserved country-specific heterogeneity and this effect is not statistically significant. Nevertheless, the maximized log-likelihood of the Butler-Moffitt model is significantly larger than that of the dynamic pooled probit model with a likelihood-ratio (LR) test statistic of 5.57. Since the parameter value under the Null hypothesis $\sigma_{\tau}=0$ lies at the boundary of the admissible parameter space, the distribution of the LRstatistic under the Null is a $\left(0.5 \chi_{(0)}^{2}+0.5 \chi_{(1)}^{2}\right)$-distribution, where $\chi_{(0)}^{2}$ represents a degenerate distribution with all its mass at origin (see, e.g., Harvey, 1989). Whence, the critical value for a significance level of $1 \%$ is the 0.98 -quantile of a $\chi_{(1)}^{2}$-distribution which equals 5.41. All in all, this evidence in favor of the random effect specification is not overwhelming. Actually, the coefficients of the explanatory variables and of the lagged dependent state are similar (after adjusting for the different normalization rules) under both specifications.

The estimated probit model with random effects assumes that $\tau_{i}$ is independent of $x_{i t}$. If this were not correct, the parameter estimates would be inconsistent. In order to check this assumption we ran the following auxiliary regression:

$$
\hat{\tau}_{i}=\psi_{0}+\bar{x}_{i \cdot}^{\prime} \psi_{1}+\zeta_{i}, \quad i=1, \ldots, n,
$$

where the vector $\bar{x}_{i}$. contains the mean values of the $x_{i t}$-variables (except for the US interest rate and the OECD growth rate) over time. The value of the $F$-statistic for the null $\psi_{1}=0$ is 1.85 with critical values of 2.03 and 1.73 for the $5 \%$ and $10 \%$ significance levels. Whence, evidence that $\tau_{i}$ might be correlated with $\bar{x}_{i}$. is inconclusive.

We now turn to the ML estimates of the dynamic random effect model that allows for serially correlated idiosyncratic errors as specified by Equations (1) and (2). This allows for all three source of serial dependence. The MLEIS estimation results based on $S=100$ EIS draws and three EIS (fixed-point) iterations are given in the left columns of Table 4. The MC (numerical) standard deviations are computed from 20 ML-EIS estimations under i.i.d. sets of CRNs. They are much smaller than the corresponding asymptotic (statistical) standard deviations indicating that the ML-EIS results are numerically very accurate.

The estimation results indicate that the inclusion of a transitory idiosyncratic error component has significant effects on the dynamic structure of the model but not on the other coefficients which remain quantitatively close to those of the Butler-Moffitt specification. The persistence parameter estimate of $\rho$ equal 0.4 and is statistically significant at the $1 \%$ level. Furthermore, the estimated coefficient $\kappa$ associated with the lagged dependent variable is now substantially smaller and not significantly different from zero. This suggests that the state dependence found under the pooled probit and the Butler-Moffitt model is spurious and the result of an improper dynamic specification of the error term (see Heckman 1981a). Since the parameter $\sigma_{\tau}$ governing the timeinvariant heterogeneity is also not statistically significant, the only source of 
serial dependence which is relevant for current account crises appears to be the transitory country-specific differences. Note that while the coefficient of $\rho$ is significant at the $1 \%$ level, the corresponding LR-statistic equals 2.57 and is not significant. Such discrepancy suggests that the lagged dependent variable in the Butler-Moffitt model acts as a proxy for serial idiosyncratic correlation.

For the purpose of comparison, the random effect model with serially correlated errors is re-estimated using the standard GHK simulator based on the same simulation sample size as used for EIS $(S=100)$. The results, which are summarized in the right columns of Table 4, reveal that the parameter estimates obtained using GHK exhibit significantly larger MC standard errors than those obtained under EIS. Moreover, while the parameter estimates for the explanatory variables are generally similar for both procedures, the estimates of the parameters governing the dynamics of current account reversals $\left(\kappa, \sigma_{\tau}\right.$, $\rho)$ are noticeably different. In particular, the ML-GHK estimates of $\sigma_{\tau}$ and $\rho$ are smaller than their ML-EIS counterparts, while that of $\kappa$ is larger. This is fully in line with the results of the MC study of Lee (1997) indicating that the ML-GHK estimator exhibits a downward bias for the persistence parameter of the idiosyncratic error as well as for the variation parameter of the unobserved heterogeneity while it is upward biased for the parameter governing the state dependence.

We now turn to the estimation results of the panel model (1), (3), and (4), allowing for unobserved random effects in both dimensions which are summarized in Table 5. The ML-EIS estimation was performed with a simulation sample size of $S=100$ and three EIS iterations. The MC standard errors reported illustrate how efficiently EIS approximates the $T+N$ integral in Equation (24). The variance parameter of the time factor and its autoregressive parameter are both significant, indicating that there are significant common dynamic time-specific effects. This empirical result, which is in line with IMF concerns (Fisher, 1998) and with several theoretical models (Corsetti et al., 1999), suggests the existence of contagion effects among developing countries. The values of the $F$-statistic for the independence test indicate that there is no evidence for correlation, neither between the time effects $\xi_{t}$ and $\bar{x}_{\cdot t}$, where $\bar{x}_{\cdot t}$ contains the mean values of the explanatory variables across countries, nor between the country effects $\tau_{i}$ and $\bar{x}_{i}$. (see Equation 32 ).

Furthermore, note that the state-dependence coefficient $\kappa$ is statistically significant, while the country-specific random effect is again not significantly different from zero. Hence, under the model with time-specific effects the lagged dependent variable seems to act (similar as under the Butler-Moffitt model) as a proxy for positive serial idiosyncratic correlation. Notice that the serial correlation associated with the factor $\xi_{t}$ is negative and is common to all countries. A comparison between the model with serially correlated idiosyncratic errors (Table 4) and that allowing random effects in both dimensions (Table 5) reveals that both are virtually observationally equivalent with very similar coefficients for all explanatory variables in $x_{i t}$.

All in all, we find under all dynamic panel models significant serial correlation characterizing the dynamics of current account crises. Furthermore, there is no evidence for persistent unobserved heterogeneity across country as a possi- 
ble source for serial dependence. Finally, the data do not allow to discriminate cleary between serially correlated idiosyncratic errors, state dependence and/or dynamic spill over effects as potential sources of serial dependence.

\section{Conclusion}

This paper uses different non-linear panel data specifications to investigate the causes and dynamics of current account reversals in low- and middle-income countries. In particular, we analyze four sources of serial persistence: a countryspecific random effect, a serially correlated transitory error component, dynamic spill over effects, and a state dependence component to control for the effect of previous events of current account reversal.

For likelihood-based estimation of panel models with country-specific random heterogeneity and serially correlated error components we propose to use Efficient Importance Sampling (EIS) which represents a Monte Carlo (MC) integration technique. The application of EIS allows for numerically very accurate and reliable ML estimation of those models. In particular, it improves significantly the numerical efficiency of GHK, which is the most frequently used MC procedure to estimate non-linear panel models with serially correlated errors.

Our empirical results show that the static pooled probit model is unable to capture the dynamic patterns of the data and that the inclusion of the lagged dependent variable significantly increases the fit of the model. In turn, that variable appears to be only a proxy for an autoregressive error structure capturing transitory unobserved differences across countries. ML-EIS estimation of a panel probit with unobserved individual heterogeneity and autocorrelated idiosyncratic errors finds that the autocorrelation coefficient of the error term is statistically significant, while both the lagged dependent and the countryspecific random effect are not. Finally, the ML-EIS estimate of a panel probit model with unobserved individual heterogeneity, as well as a correlated timespecific effects reveals that the time-specific effects indicative of contagion effects among developing countries are siginificant. We found, however, that the model with unobserved individual heterogeneity and serially correlated idiosyncratic errors and that with random country-specific and correlated time-effects are virtually observationally equivalent with very similar coefficients for all explanatory variables.

The empirical results of both models suggest that countries with high current account imbalances, low foreign reserves, a small fraction of concessional debt, and unfavorable terms of trades are more likely to experience a current account reversal. 


\section{References}

Baldwin, R., Krugman, P., 1989. Persistent trade effects of large exchange rate schocks. The Quaterly Journal of Economics 54, 635-654.

Baltagi, B., 2005. Econometric Analysis of Panel Data. John Wiley \& Sons.

Butler, J.S., Moffitt, R., 1982. A computationally efficient quadrature procedure for the one-factor multinomial probit model. Econometrica 50, 761-764.

Calvo, G., Izquierdo, A., Mejia, L.F., 2004. On the empirics of sudden stops: the relevance of balance-sheet effects. Working paper. Inter-American Development Bank.

Corsetti, G., Pesenti, P., Roubini, N., Tille, C. 1999. Competitive devaluations: a welfare-based approach. NBER-Working Paper No 6889.

DeGroot, M.H., 1970. Optimal Statistical Decisions. McGraw-Hill.

Devroye, L., 1986. Non-Uniform Random Variate Generation. Springer-Verlag.

Dixit, A., 1992. Investment and Hysteresis. The Journal of Economic Perspectives 6, $107-132$.

Dornbusch, R., Park, Y.C., Claessens, S., 2000. Contagion: Understanding how it spreads. World Bank Research Observer 15, 177-197.

Edwards, S., 2004a. Financial openness, sudden stops and current account reversals. American Economic Review 2, 59-64.

Edwards, S., 2004b. Thirty years of current account imbalances, current account reversals, and sudden stops. NBER-Working Paper No. 10276.

Edwards, S., Rigobon, R. 2002. Currency crises and contagion: an introduction. Journal of Development Economics 69, 307-313.

Eichengreen, B., Rose, A., Wyplosz 1995. Exchange rate mayhem: the antecedents and aftermath of speculative attacks. Economic Policy 21, 249-312.

Falcetti, E., Tudela, M., 2006. Modelling currency crises in emerging markets: a dynamic probit model with unobserved heterogeneity and autocorrelated errors. Oxford Bulletin of Economics and Statistics 68, 445-471.

Fisher, S., 1998. The IMF and the asian crisis. Los Angeles, March 20.

Frankel, J., Rose, A., 1996. Currency crashes in emerging markets: an empirical treatment. Board of Governors of the Federal Reserve System Oxford, International Finance Diskussion Papers 534, 1-28.

Geweke, J., 1991. Efficient simulation from the multivariate normal and student$\mathrm{t}$ distributions subject to linear constraints. Computer Science and Statistics: Proceedings of the Twenty-Third Symposium on the Interface, 571-578.

Geweke, J., Keane, M., 2001. Computationally intensive methods for integration in econometrics. In Heckman, J., Leamer, E., Handbook of Econometrics 5, Chapter 56. Elsevier.

Greene, W., 2003. Econometrics Analysis. Prentice Hall, Englewood Cliffs.

Greene, W., 2004. Convenient estimators for the panel probit model: further results. Empirical Economics 29, 21-47.

Hajivassiliou, V., 1990. Smooth simulation estimation of panel data LDV models. Mimeo. Yale University. 
Harvey, A., 1989. Forecasting, Structural Time Series Models and Kalman Filter. Cambridge University Press.

Heckman, J., 1981a. Statistical models for discrete panel data. In Manski, C.F., McFadden, D., Structural Analysis of Discrete Data with Econometric Applications. The MIT Press.

Heckman, J., 1981b. The incidental parameter problem and the problem of initial conditions in estimating a discrete time-discrete data stochastic process. In Manski, C.F., McFadden, D., Structural Analysis of Discrete Data with Econometric Applications. The MIT Press.

Hyslop, D., 1999. State Dependence, serial correlation and heterogeneity in intertemporal labor force participation of married women. Econometrica 67, 1255-1294.

Keane, M., 1994. A computationally practical simulation estimator for panel data. Econometrica 62, 95-116.

Lee, L-F., 1997. Simulated maximun likelihood estimation of dynamic discrete choice models - some Monte Carlo results. Journal of Econometrics 82, 1-35.

Lehmann, E.L., 1986. Testing Statistical Hypotheses. John Wiley \& Sons.

Liesenfeld, R., Richard, J.F., 2007. Simulation techniques for panels: Efficient importance sampling. manuscript, University of Kiel, Dept. of Economics. (to appear in: Matyas, L., Sevestre, P., The Economterics of Panel Data (3rd ed). Kluwer Academic Publishers.)

Milesi-Ferretti, G.M., Razin, A., 1996. Current account sustainability: Selected east asian and latin american experiences. NBER-Working Paper No. 5791.

Milesi-Ferretti, G.M., Razin, A., 1998. Sharp reductions in current account deficits: An empirical analysis. European Economic Review 42, 897-908.

Milesi-Ferretti, G.M., Razin, A., 2000. Current account reversals and currency crisis: empirical regularities. In Krugman, P., Currency Crises. University of Chicago Press.

Obstfeld, M., Rogoff, K. 1996. Foundations of International Macroeconomics. The MIT Press.

Obstfeld, M., Rogoff, K., 2004. The unsustainable US current account position revisited. NBER-Working paper No. 10869.

Richard, J.-F., 1977. Bayesian analysis of the regression model when the disturbances are generated by an autoregressive process. In Aykac, A., Brumat, C., New developments in the application of Bayesian methods. North Holland, Amsterdam.

Richard, J.-F., Zhang, W., 2007. Efficient high-dimensional importance sampling. Forthcoming in: Journal of Econometrics.

Stern, S., 1997. Simulation-based estimation. Journal of Economic Literature 35, 2006-2039.

Train, K.E., 2003. Discrete Choice Methods with Simulation. Cambridge University Press.

Zhang, W., Lee, L.-F., 2004. Simulation estimation of dynamic discrete choice panel models with accelerated importance samplers. Econometrics Journal 7, 120-142. 
Table 2. ML-estimates of the pooled probit model

\begin{tabular}{|c|c|c|c|c|}
\hline \multirow[b]{2}{*}{ Variable } & \multicolumn{2}{|c|}{ Static Model } & \multicolumn{2}{|c|}{ Dynamic Model } \\
\hline & Estimate & Asy. s.e. & Estimate & Asy. s.e. \\
\hline Constant & $-1.993^{* * *}$ & 0.474 & $-1.955^{* * *}$ & 0.493 \\
\hline AVGCA & $-0.060^{* * *}$ & 0.012 & $-0.060^{* * *}$ & 0.012 \\
\hline AVGGROW & 0.008 & 0.021 & 0.009 & 0.021 \\
\hline AVGINV & -0.002 & 0.010 & 0.001 & 0.011 \\
\hline AVGTT & -0.108 & 0.066 & -0.109 & 0.069 \\
\hline GOV & $0.026^{* *}$ & 0.012 & 0.018 & 0.012 \\
\hline OT & -0.011 & 0.010 & -0.011 & 0.010 \\
\hline OPEN & -0.058 & 0.087 & -0.085 & 0.090 \\
\hline USINT & 0.108 & 0.073 & 0.107 & 0.075 \\
\hline GROWOECD & 0.084 & 0.086 & 0.042 & 0.090 \\
\hline INTPAY & 0.024 & 0.029 & 0.021 & 0.030 \\
\hline RES & $-0.074^{* *}$ & 0.030 & $-0.074^{* *}$ & 0.030 \\
\hline CONCDEB & $-0.165^{* *}$ & 0.068 & $-0.152^{* *}$ & 0.071 \\
\hline$\kappa$ & & & $0.981^{* * *}$ & 0.158 \\
\hline Log-likelihood & -276.13 & & -257.26 & \\
\hline
\end{tabular}

Note: The estimated model is given by Equation (1) assuming that the errors are independent across countries and time. The asymptotic standard errors are calculated as the square root of the diagonal elements of the inverse Hessian. ${ }^{*},{ }^{* *}$, and ${ }^{* * *}$ indicates statistical significance at the $10 \%, 5 \%$ and $1 \%$ significance level. 
Table 3. ML-estimates of the dynamic Butler-Moffitt random effect model

\begin{tabular}{lcr}
\hline \hline Variable & Estimate & Asy. s.e. \\
\hline Constant & $-1.880^{* * *}$ & 0.534 \\
AVGCA & $-0.064^{* * *}$ & 0.015 \\
AVGGROW & 0.010 & 0.021 \\
AVGINV & -0.0001 & 0.011 \\
AVGTT & -0.122 & 0.084 \\
GOV & 0.018 & 0.012 \\
OT & -0.011 & 0.011 \\
OPEN & -0.069 & 0.093 \\
USINT & 0.083 & 0.075 \\
GROWOECD & 0.073 & 0.090 \\
INTPAY & 0.014 & 0.031 \\
RES & $-0.073^{* *}$ & 0.035 \\
CONCDEB & $-0.159^{* *}$ & 0.078 \\
$\kappa$ & $0.982^{* * *}$ & 0.154 \\
$\sigma_{\tau}$ & 0.162 & 0.210 \\
$\sigma_{e}$ & 1.013 & \\
Log-likelihood & -254.47 & \\
LR-statistic for $\mathrm{H}_{0}: \sigma_{\tau}=0$ & 5.57 & \\
$F$-statistic for exogeneity & 1.85 & \\
\hline
\end{tabular}

Note: The estimated model is given by Equation (1) and (1). The ML-estimation is based on a Gauss-Hermite quadrature using 20 nodes. The asymptotic standard errors are calculated as the square root of the diagonal elements of the inverse Hessian. ${ }^{*},{ }^{* *}$, and ${ }^{* * *}$ indicates statistical significance at the $10 \%, 5 \%$ and $1 \%$ significance level. The $1 \%$ and $5 \%$ percent critical values of the LR-statistic for $\mathrm{H}_{0}: \sigma_{\tau}=0$ are 5.41 and 2.71 . The $1 \%$ and $5 \%$ percent critical values of the $F$-statistic for exogeneity are 2.71 and 2.03 . 
Table 4. ML-estimates of the dynamic random effect model with serially correlated idiosyncratic errors

\begin{tabular}{|c|c|c|c|c|c|c|}
\hline \multirow[b]{2}{*}{ Variable } & \multicolumn{3}{|c|}{ ML-EIS } & \multicolumn{3}{|c|}{ ML-GHK } \\
\hline & Est. & $\begin{array}{l}\text { Asy. } \\
\text { s.e. }\end{array}$ & $\begin{array}{l}\text { MC } \\
\text { s.e. }\end{array}$ & Est. & $\begin{array}{l}\text { Asy. } \\
\text { s.e. }\end{array}$ & $\begin{array}{l}\text { MC } \\
\text { s.e. }\end{array}$ \\
\hline Constant & $-1.623^{* * *}$ & 0.224 & 0.0074 & $-1.752^{* * *}$ & 0.526 & 0.1015 \\
\hline AVGCA & $-0.077^{* * *}$ & 0.018 & 0.0006 & $-0.074^{* * *}$ & 0.017 & 0.0028 \\
\hline AVGGROW & 0.006 & 0.028 & 0.0003 & 0.007 & 0.026 & 0.0009 \\
\hline AVGINV & 0.004 & 0.016 & 0.0003 & 0.004 & 0.015 & 0.0012 \\
\hline AVGTT & $-0.189^{*}$ & 0.103 & 0.0030 & $-0.171^{*}$ & 0.094 & 0.0170 \\
\hline GOV & 0.017 & 0.016 & 0.0001 & 0.018 & 0.015 & 0.0006 \\
\hline OT & -0.010 & 0.014 & $>0.0001$ & -0.010 & 0.013 & 0.0007 \\
\hline OPEN & -0.123 & 0.122 & 0.0029 & -0.116 & 0.118 & 0.0164 \\
\hline USINT & 0.093 & 0.082 & 0.0009 & 0.098 & 0.082 & 0.0074 \\
\hline GROWOECD & 0.052 & 0.096 & 0.0011 & 0.054 & 0.093 & 0.0070 \\
\hline INTPAY & 0.031 & 0.040 & 0.0007 & 0.030 & 0.038 & 0.0037 \\
\hline RES & $-0.109^{* *}$ & 0.047 & 0.0018 & $-0.103^{* *}$ & 0.047 & 0.0100 \\
\hline CONCDEB & $-0.210^{* *}$ & 0.080 & 0.0024 & $-0.199^{* *}$ & 0.093 & 0.0152 \\
\hline$\kappa$ & 0.440 & 0.284 & 0.0173 & $0.486^{*}$ & 0.259 & 0.0764 \\
\hline$\sigma_{\tau}$ & 0.199 & 0.794 & 0.0048 & $0.078^{*}$ & 0.051 & 1.9035 \\
\hline$\delta$ & $0.404^{* * *}$ & 0.150 & 0.0136 & $0.376^{* *}$ & 0.175 & 0.0615 \\
\hline$\sigma_{e}$ & 1.111 & & & 1.082 & & \\
\hline Log- & & & & & & \\
\hline $\begin{array}{l}\text { likelihood } \\
\text { LR-stat. for }\end{array}$ & -253.19 & & 0.0356 & -253.20 & & 0.3356 \\
\hline $\mathrm{H}_{0}: \rho=0$ & 2.57 & & & 2.55 & & \\
\hline $\begin{array}{l}F \text {-stat. for } \\
\text { exogeneity }\end{array}$ & 1.32 & & & & & \\
\hline
\end{tabular}

Note: The estimated model is given by Equation (1) and (2). The ML-EIS and ML-GHK estimation are based on a MC sample size of $S=100$. The EIS simulator is based on three EIS iterations. The asymptotic standard errors are calculated as the square root of the diagonal elements of the inverse Hessian and the MC standard errors from 20 replications of the ML-EIS and ML-GHK estimation. ${ }^{*},{ }^{* *}$, and *** indicates statistical significance at the $10 \%, 5 \%$ and $1 \%$ significance level. The $1 \%$ and $5 \%$ percent critical values of the LR-statistic for $\mathrm{H}_{0}: \rho=0$ are 6.03 and 3.84 . The $1 \%$ and $5 \%$ percent critical values of the $F$-statistic for exogeneity are 2.71 and 2.03 . 
Table 5. ML-estimates of the dynamic model with random country-specific and time effects

\begin{tabular}{lcrr}
\hline \hline Variable & Estimate & Asy. s.e. & MC. s.e. \\
\hline Constant & $-1.967^{* * *}$ & 0.677 & 0.0008 \\
AVGCA & $-0.064^{* * *}$ & 0.014 & 0.0001 \\
AVGGROW & 0.013 & 0.022 & $>0.0001$ \\
AVGINV & -0.001 & 0.011 & $>0.0001$ \\
AVGTT & -0.122 & 0.075 & 0.0005 \\
GOV & 0.018 & 0.012 & $>0.0001$ \\
OT & -0.010 & 0.011 & $>0.0001$ \\
OPEN & -0.065 & 0.095 & 0.0002 \\
USINT & 0.070 & 0.071 & 0.0002 \\
GROWOECD & 0.113 & 0.097 & 0.0001 \\
INTPAY & 0.011 & 0.032 & $>0.0001$ \\
RES & $-0.073^{* *}$ & 0.035 & 0.0002 \\
CONCDEB & $-0.163^{* *}$ & 0.074 & 0.0003 \\
$\kappa$ & $1.013^{* * *}$ & 0.139 & 0.0004 \\
$\sigma_{\tau}$ & 0.154 & 0.201 & 0.0028 \\
$\delta$ & $-0.888^{* * *}$ & 0.041 & 0.0003 \\
$\sigma_{\xi}$ & $0.089^{* *}$ & 0.048 & 0.0002 \\
$\sigma_{e}$ & 1.030 & & \\
Log-likelihood & -253.1287 & & 0.0052 \\
$F$-stat. for exogeneity $\left(\tau_{i}\right)$ & 0.86 & & \\
$F$-stat. for exogeneity $\left(\xi_{t}\right)$ & 0.51 & & \\
\hline
\end{tabular}

Note: The estimated model is given by Equation (1), (3), and (4). The ML-EIS estimation is based on a MC sample size of $S=100$ and three EIS iterations. The asymptotic standard errors are calculated as the square root of the diagonal elements of the inverse Hessian and the MC standard errors from 20 replications of the ML-EIS estimation. ${ }^{*},{ }^{* *}$, and *** indicates statistical significance at the $10 \%, 5 \%$ and $1 \%$ significance level. The $1 \%$ and $5 \%$ percent critical values of the $F$-statistic for exogeneity in the regression for $\tau_{i}$ are given by 2.71 and 2.03 and in the regression for $\xi_{t}$ by 7.72 and 4.00 , respectively. 